\title{
Screening a Solitary Dilated Duct in the Breast: A Pictorial Essay
}

\author{
Veronica Jorge Ayres (iD' \\ Luciana Costa Ramalho' \\ Sheila Cristina Lordelo Wludarski ${ }^{2}$ \\ Eduardo de Faria Castro \\ Fleury (iD) \\ 'Department of Radiology of Instituto \\ Brasileiro do Controle do Câncer, São \\ Paulo, SP, 03 I02-002, Brazil; '2Department \\ of Pathology of Instituto Hermes Pardini \\ SA, Belo Horizonte, MG, Brazil
}

Correspondence: Veronica Jorge Ayres Instituto Brasileiro do Controle do

Câncer, Av Alcântara Machado, 2576, São

Paulo, 03I02-002, SP, Brazil

Tel + 55 II 3474-4222

Email ayresve@gmail.com

\begin{abstract}
A solitary dilated duct visualized by mammography is a rare event. According to the latest edition of BI-RADS ${ }^{\circledR}$ it is classified as category 4. This series of cases shows complementary ultrasound of a solitary dilated duct can reduce false-positive results on mammography.
\end{abstract}

Keywords: breast, duct, mammography, ultrasound

\section{Background}

Mammographic solitary dilated duct (SDD) is described as a rare screening finding. According to BI-RADS ${ }^{\circledR}$ 5th edition, SDD is defined as a tubular or branched structure in the retro-areolar region of the breast. ${ }^{1}$

Previously classified only as an associated finding and not related to breast cancer, ${ }^{2}$ since the BI-RADS ${ }^{\circledR}$ last edition, SDD has been classified as category 4. BI-RADS ${ }^{\circledR}$ recommends biopsy of these lesions visualized by mammography, according to Chang et al. ${ }^{3}$

In a recent study, our group evaluated 94 solitary dilated ducts visualized by mammography with complementary target ultrasound. Of these, no cancer lesions were observed. The most relevant histological finding when associated with an ultrasound abnormality was papillary lesion. ${ }^{4}$ This series of cases of a complementary ultrasound of mammographic solitary dilated duct shows that target ultrasound can reduce false-positive results on mammography.

\section{Materials and Methods}

From March 2016 to March 2017, a prospective study was carried out to assess solitary dilated ducts visualized by mammography in a reference hospital for the diagnosis, treatment, and follow-up of breast cancer. A total of 9035 screening and diagnostic mammograms were evaluated. Bilateral digital two-dimensional (2D) mammograms were performed at one cranio-caudal and medio-lateral oblique incidence of each breast without complementary magnification or compression specifically for SDD evaluation. When SDD was characterized by mammography, targeted ultrasound (US) for diagnostic complementation was performed, followed by percutaneous biopsy, if necessary. The assessment was performed by physicians with 2 or 3 years of experience in mammogram evaluation and validated by a specialist with 15 years of experience in breast imaging. This study was approved by the Local Research Ethics Committee of Instituto Brasileiro de Controle do Câncer, São Paulo, Brazil. All patients who were evaluated by targeted US accepted the informed consent form. From this 
database, we selected eight different cases, with some similar radiological features, but with different histology.

\section{Results}

\section{False-Positive Mammography}

Occasionally, a solitary dilated duct visualized by mammography cannot be confirmed by targeted US. Prior surgery or other mammary parenchyma changes can mimetize a solitary dilated duct at mammography.

In this case, a 78-year-old woman with no breast cancer risk factors and no history of breast surgery had a screening mammogram (Figure 1). Left mammogram of the breast was almost entirely fatty and can identify a solitary dilated duct with high density (blue arrow) at the cranio-caudal (CC) mammogram view (a) and medio-lateral oblique (MLO) mammogram view (b). Targeted US shows no findings at the retro-areolar area (c).

\section{Solitary Dilated Duct Visualized by Mammography Associated with \\ Calcifications}

In our study, only cases of calcifications were included as associated findings and not as main findings, so all suspicious calcifications associated with SDD were excluded. This is an example that we value SDD as a primary finding and round calcifications as a secondary finding. ${ }^{4}$ In this case, a 56-year-old woman with no breast cancer risk factors and no history of breast surgery had a screening mammogram (Figure 2). In the left mammogram there were scattered areas of fibroglandular density and it can identify medium density SDD (blue arrow) at the cranio-caudal (CC) mammogram view (a) and medio-lateral oblique (MLO) mammogram view (b) with round calcifications. Targeted US solitary dilated duct shows intraductal heterogeneous intraductal content (blue arrow) (c); H\&E staining (x10) shows papilloma, confirmed by lumpectomy surgery (d).

\section{Solitary Dilated Duct Visualized by Mammography and Homogeneous Duct Content on Targeted US}

In this case a 62-year-old woman with no breast cancer risk factors and no history of breast surgery had a screening mammogram (Figure 3). Left mammogram showed there was scattered areas of fibroglandular density and can identify medium density SDD (green arrow) at the cranio-caudal (CC) mammogram view (a) and medio-lateral oblique (MLO) mammogram view (b). Targeted US solitary dilated duct shows anechoic intraductal content (green arrow) (c); H\&E staining (x10) shows ductal ectasia (d).
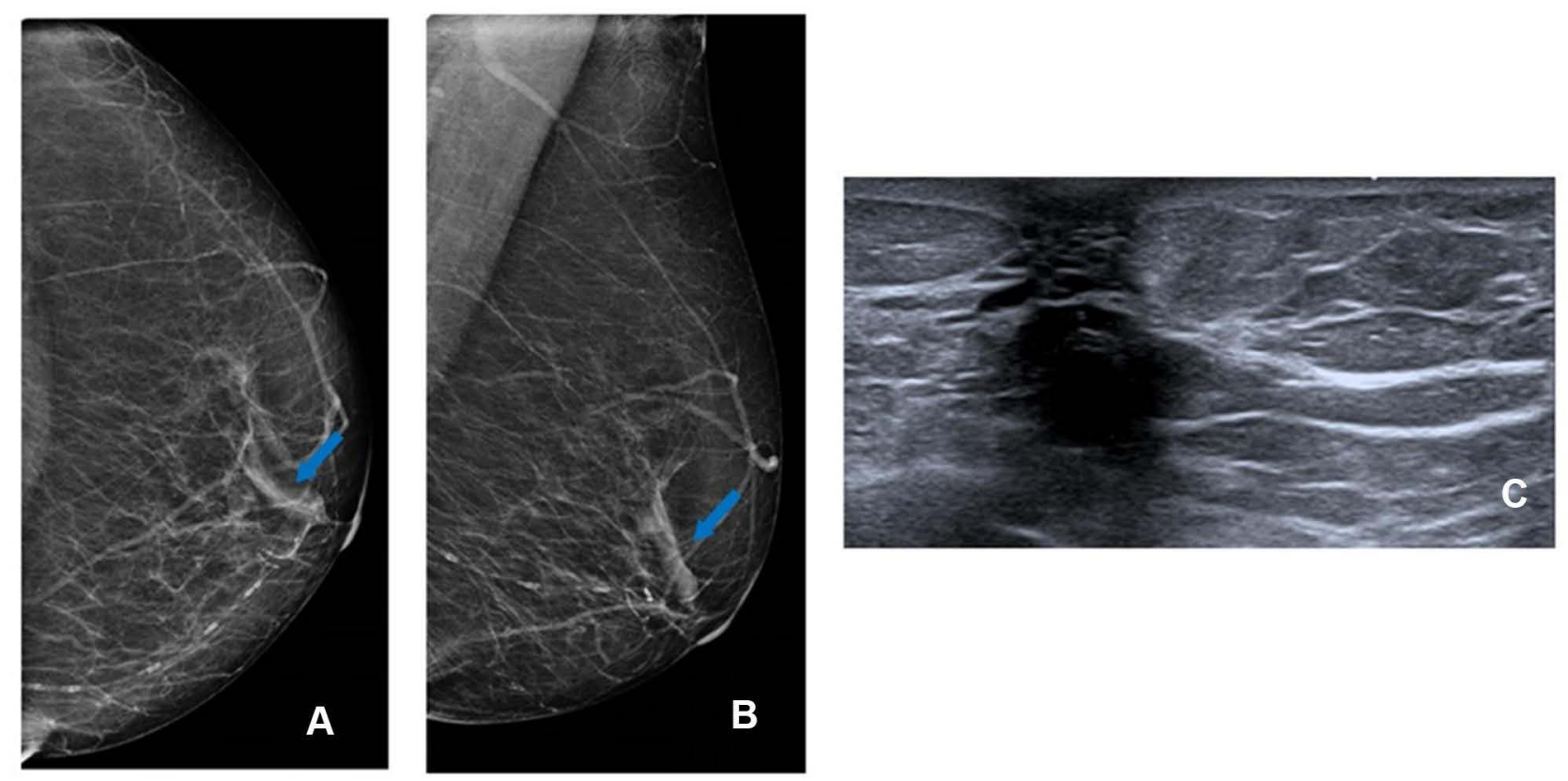

Figure I False-positive mammography. Solitary dilated duct (blue arrow) at left cranio-caudal (CC) mammogram view (A) and at medio-lateral oblique (MLO) mammogram view (B); ultrasound (US) shows no findings at retro-areolar area (C). 

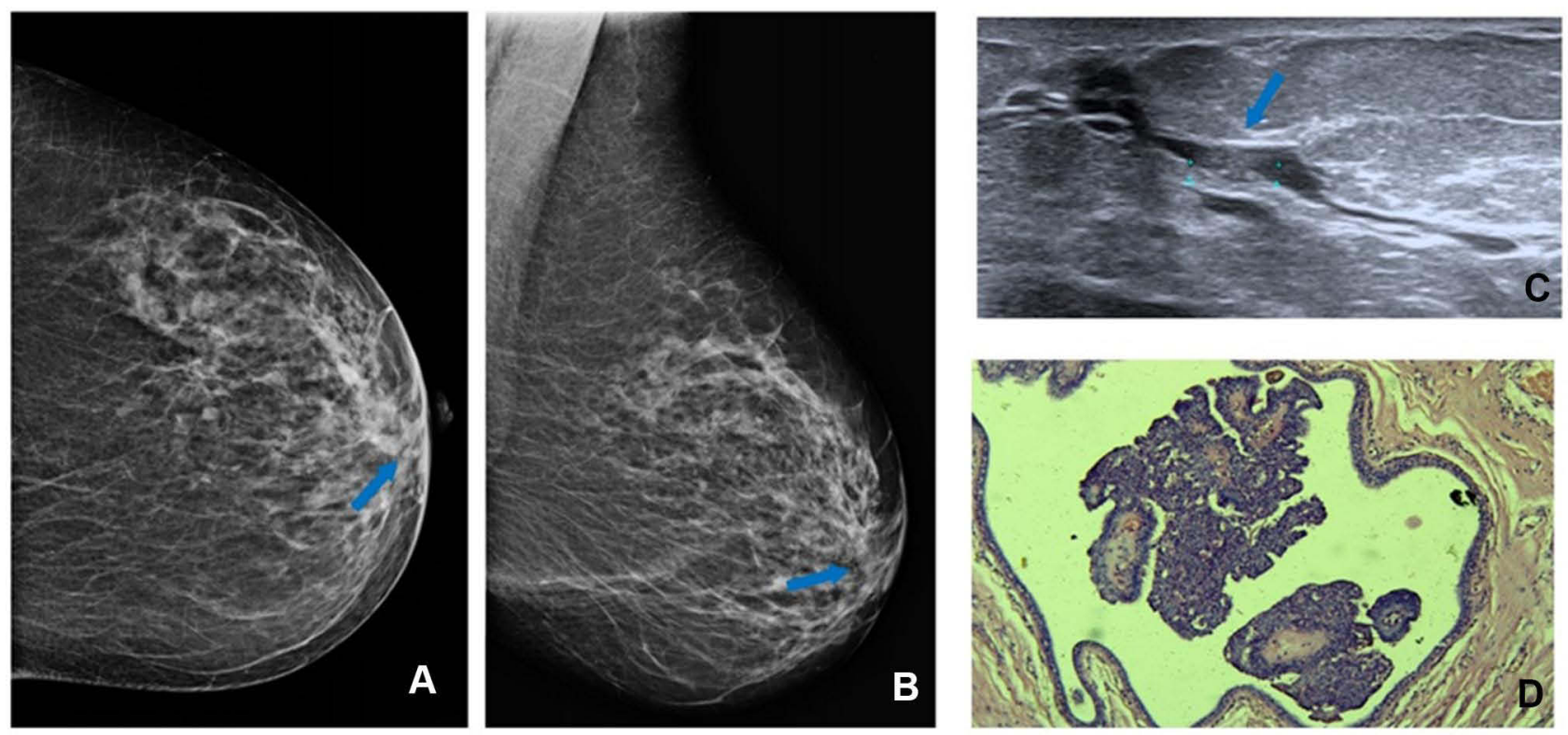

Figure 2 Solitary dilated duct with round calcifications (blue arrow) at cranio-caudal (CC) (A) and at medio-lateral oblique (MLO) (B) left mammogram view; ultrasound (US) shows intraductal heterogeneous intraductal content (blue arrow) (C). H\&E staining (xI0) shows papilloma (D).
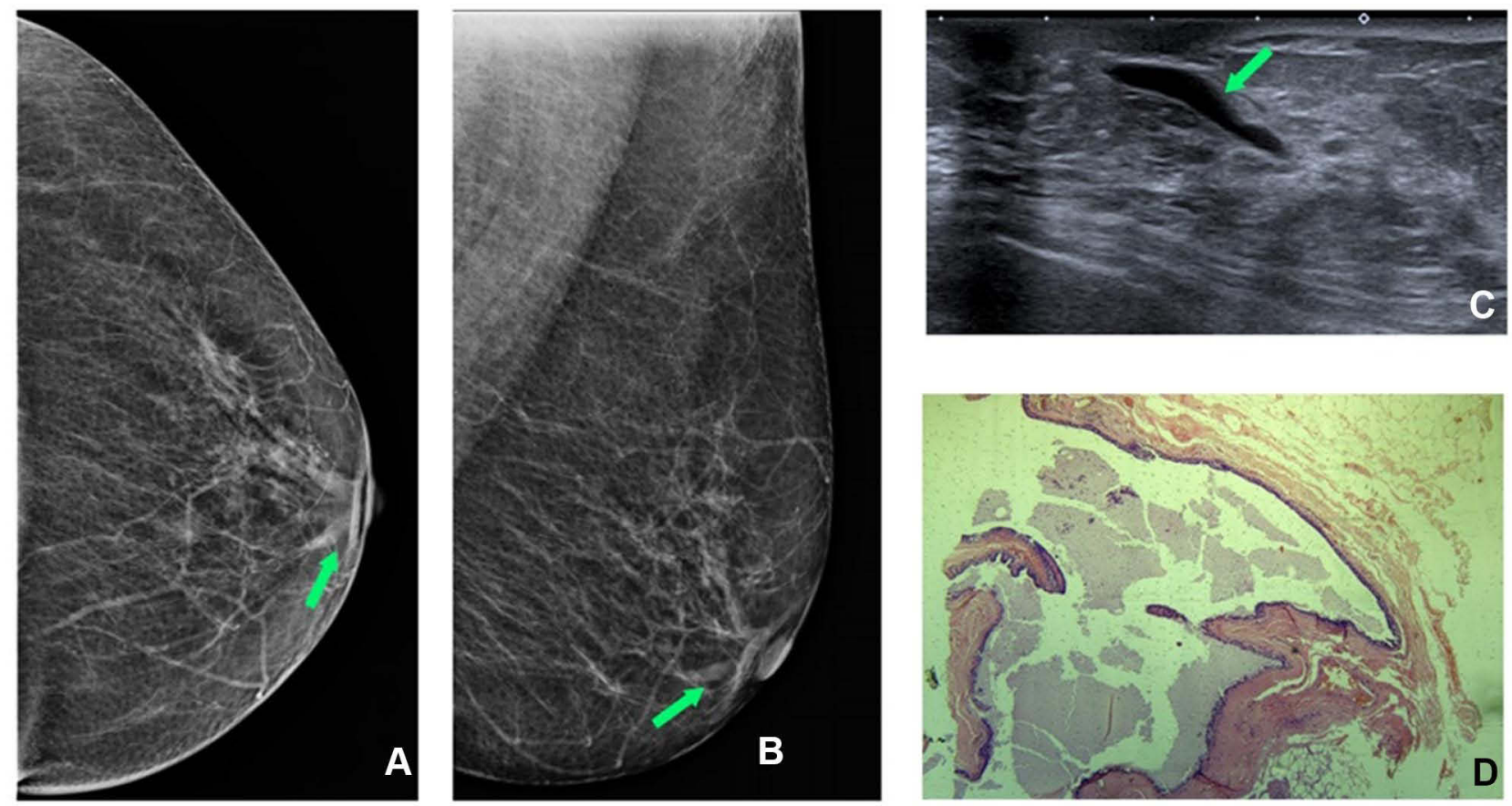

Figure 3 Left mammogram SDD (green arrow) at cranio-caudal (CC) (A) and medio-lateral oblique (MLO) (B) mammogram view. Targeted US shows anechoic intraductal content (green arrow) (C); (D) H\&E staining (x/0) shows ductal ectasia.

Ductal ectasia is mostly common finding in targeted US solitary dilated duct visualized by mammography ${ }^{4}$ and as a finding of screening tests and in patients with nipple flow. Ductal ectasia presents an anechogenic or hypoechogenic intraductal content without malignancy suspect. ${ }^{5}$

\section{Solitary Dilated Duct Visualized by} Mammography and Intraductal Heterogeneous Content on Targeted US

In this case, a 50-year-old woman with no breast cancer risk factors and no history of breast surgery had a 
screening mammogram (Figure 4). Right mammogram shows the breast heterogeneously dense, which may obscure small masses, and it can identify medium density SDD (blue arrow) at the cranio-caudal (CC) mammogram view (a) and medio-lateral oblique (MLO) mammogram view (b). Targeted US solitary dilated duct shows intraductal heterogeneous intraductal content (blue arrow) (c); H\&E staining (x10) shows papilloma (d), confirmed by immunohistochemistry evaluation.

Intraductal papilloma is the most common papillary lesion and it can be classified as central or peripheral, depending on the mammary localization. The papilloma related to $\mathrm{SDD}$ is the central one and shows a single intraductal lesion. ${ }^{6}$

Another lesion that can also present as heterogeneous intraductal content at targeted US is fibrocystic changes . In this case, a 64-year-old woman with no breast cancer risk factors and no history of breast surgery had a screening mammogram (Figure 5). Left mammogram shows the breast heterogeneously dense, which may obscure small masses, and it can identify medium density SDD (green arrow) at the cranio-caudal (CC) mammogram view (a) and medio-lateral oblique (MLO) mammogram view (b). Targeted US solitary dilated duct shows intraductal heterogeneous intraductal content (green arrow) (c); H\&E staining (x10) shows fibrocystic changes (d).
Fibrosclerosis was another histological finding related to a dilated duct on ultrasonography with heterogeneous content, as we can characterize in this case. A 53-year-old woman with no breast cancer risk factors and no history of breast surgery had a screening mammogram (Figure 6). Right mammogram shows the breast was almost entirely fatty and we can identify medium density SDD (yellow arrow) at the cranio-caudal (CC) mammogram view (a) and medio-lateral oblique (MLO) mammogram view (b). Targeted US solitary dilated duct shows intraductal heterogeneous intraductal content (yellow arrow) (c); H\&E staining (x10) shows stromal fibrosclerosis (d).

\section{Solitary Dilated Duct Visualized by Mammography and Elastography in Intraductal Mass on Targeted US}

Since the last edition of BI-RADS ${ }^{\circledR}$, ultrasound with elastography has been a tool in the diagnosis of breast lesions. ${ }^{1}$ This method allows for the delimitation of an area of interest (ROI) and it is assumed that more rigid areas are more suspected of malignancy. One of the types of elastography (strain elastography) allows, through the manual compression of the studied tissue, the representation in colors of areas according to greater or lesser rigidity about the adjacent tissue. ${ }^{7}$ In these cases, strain elastography was an auxiliary method in the indication of
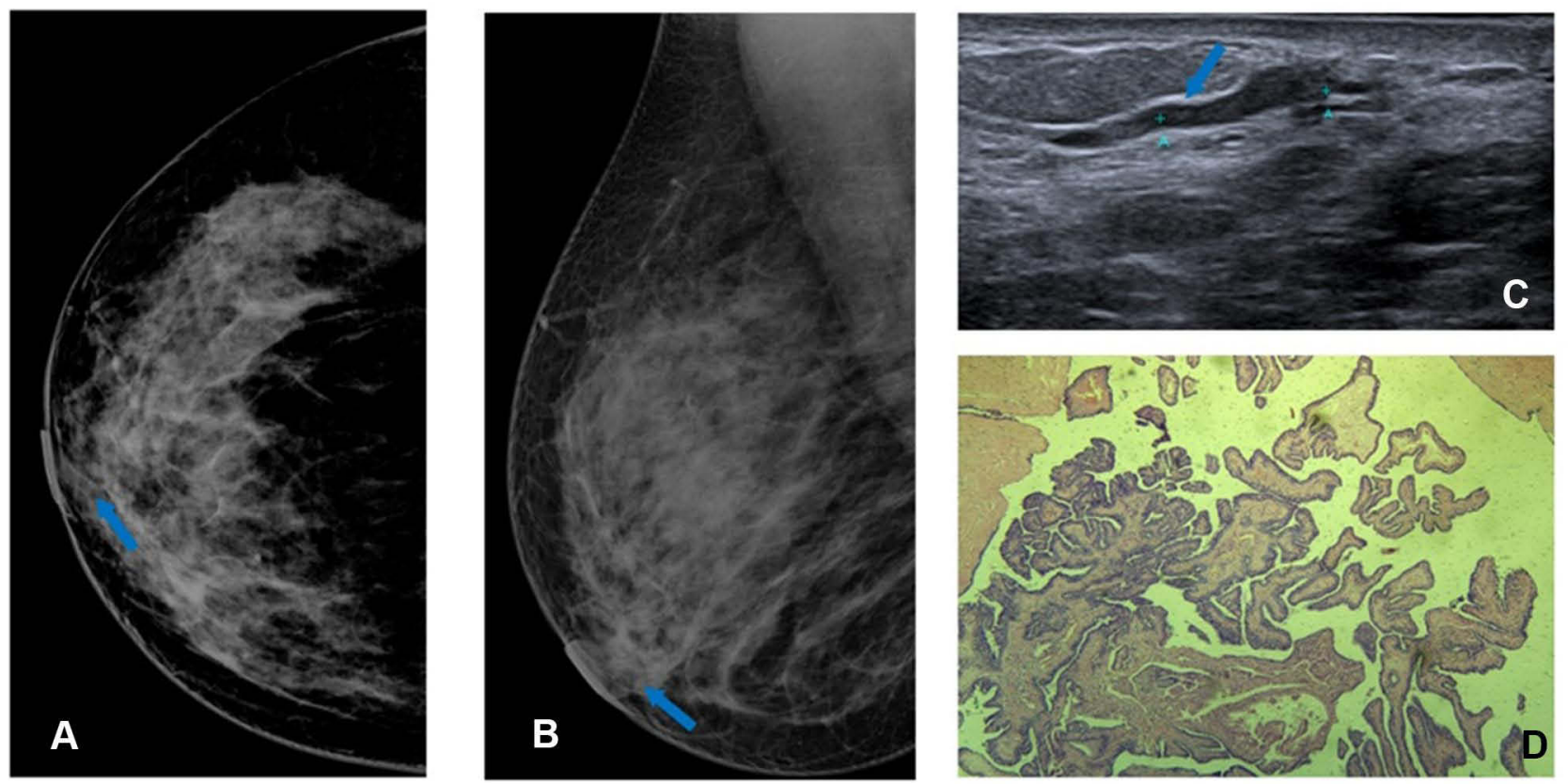

Figure 4 Right mammogram shows SDD (blue arrow) at cranio-caudal (CC) (A) and medio-lateral oblique (MLO) (B) mammogram view . Targeted US shows intraductal heterogeneous intraductal content (blue arrow) (C); H\&E staining ( $x 10)$ shows papilloma (D). 

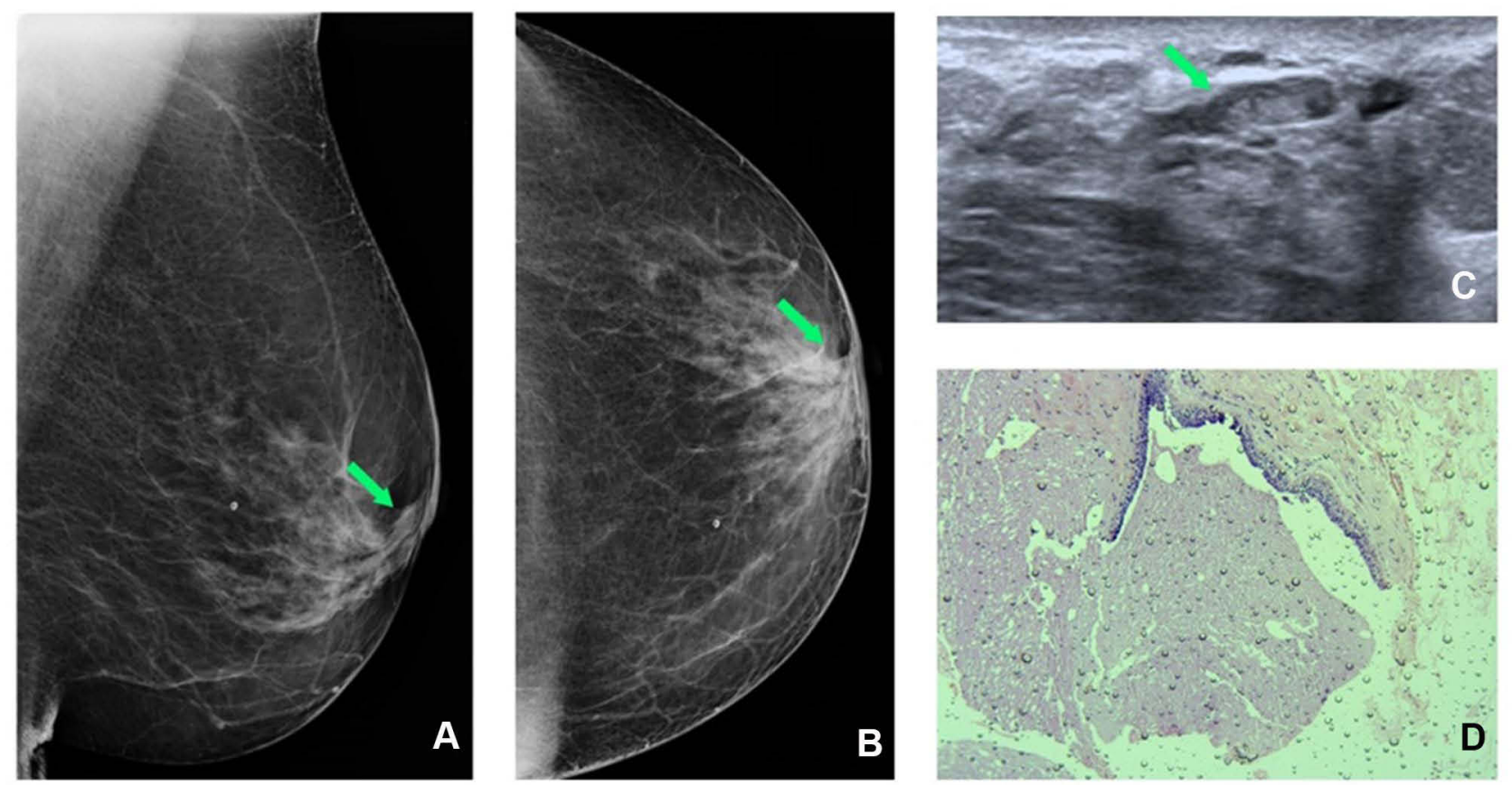

$\mathbf{B}$

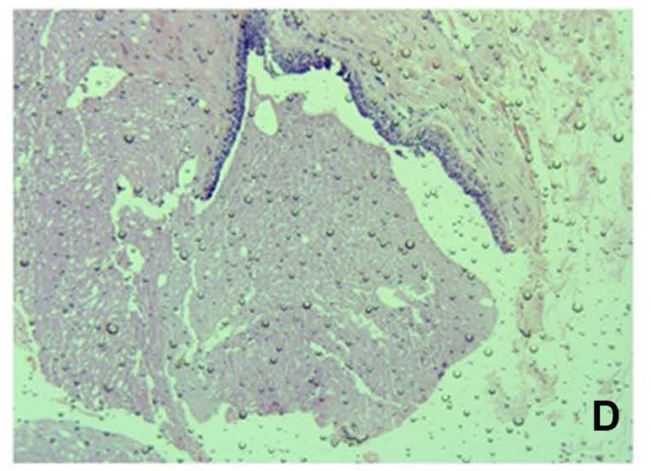

Figure 5 Left mammogram SDD (green arrow) at cranio-caudal (CC) (A) and medio-lateral oblique (MLO) (B) mammogram view. Targeted US shows intraductal heterogeneous intraductal content (green arrow) (C); H\&E staining ( $x \mid 0)$ shows fibrocystic changes (D).
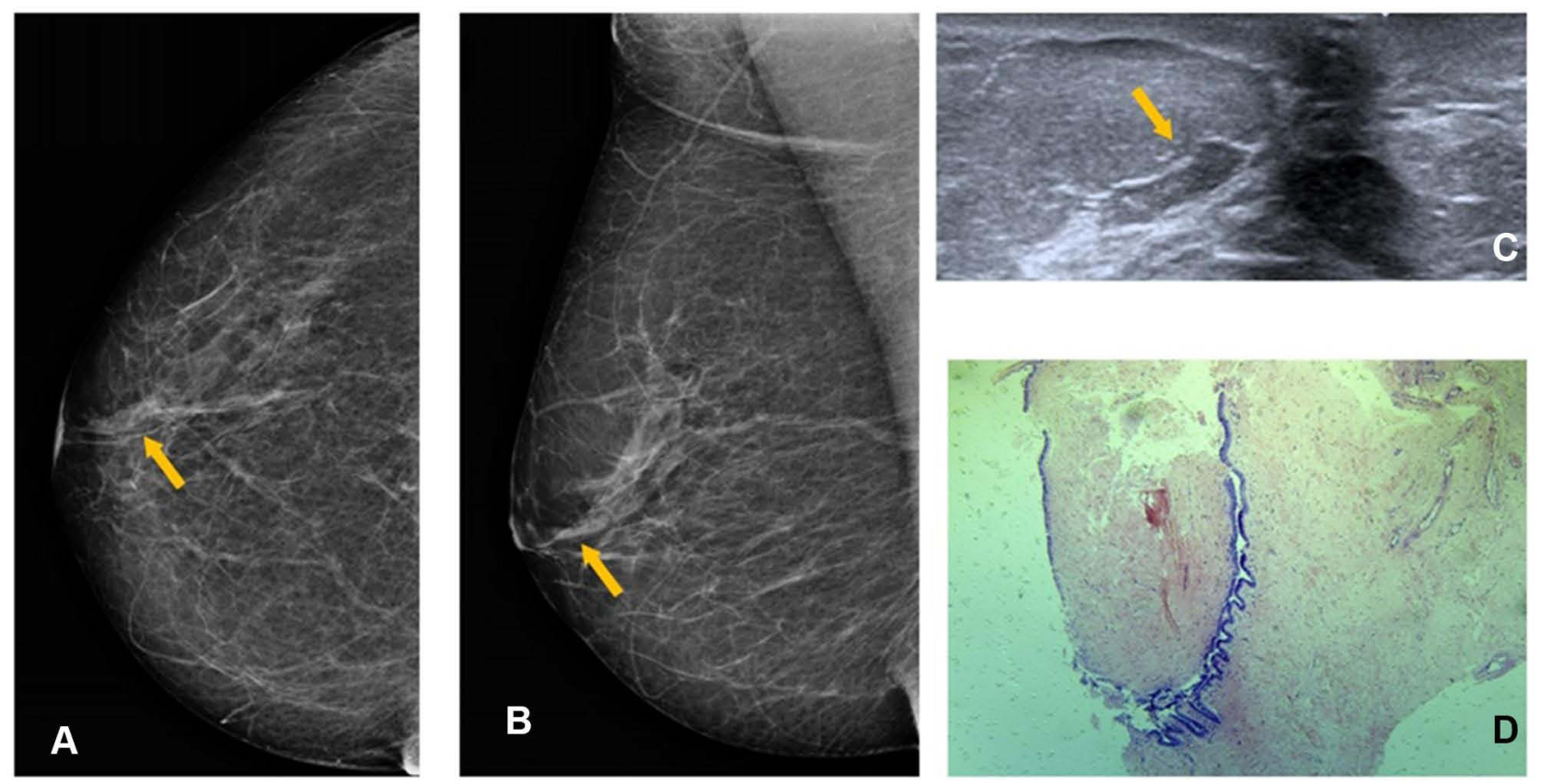

Figure 6 Right mammogram shows SDD (yellow arrow) at cranio-caudal (CC) (A) and medio-lateral oblique (MLO)(B) mammogram view. Targeted US shows intraductal heterogeneous intraductal content (yellow arrow) (C); H\&E staining $(x \mid 0)$ shows stromal fibrosclerosis (D).

percutaneous biopsy. In this case, a 45-year-old woman with no breast cancer risk factors and no history of breast surgery had a screening mammogram (Figure 7). Left mammogram shows the breast heterogeneously dense, which may obscure small masses, and it can identify medium density SDD (yellow arrow) at the cranio-caudal (CC) mammogram view (a) and medio-lateral oblique (MLO) mammogram view (b). Targeted US shows 

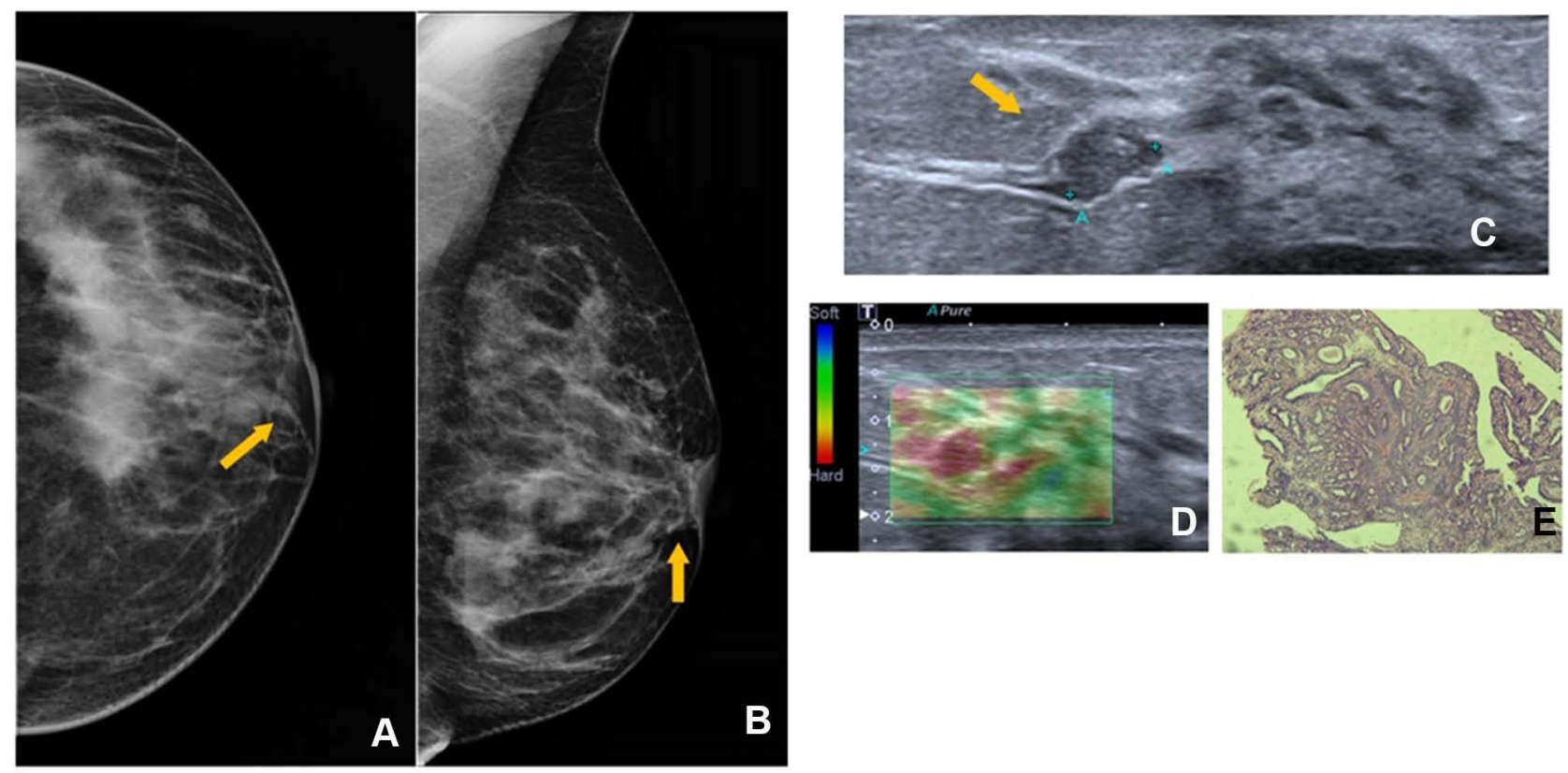

Figure 7 Left mammogram SDD (yellow arrow) at cranio-caudal (CC) (A) and medio-lateral oblique (MLO)(B) mammogram view. Targeted US shows intraductal mass (yellow arrow) (C) and high elastography (D); H\&E staining (xI0) shows sclerosing papilloma (E).

intraductal mass (c) and high elastography (d); H\&E staining (x10) shows sclerosing papilloma (e), confirmed by immunohistochemistry evaluation.

The following case demonstrates another benign lesion had rigid elastography. A 69-year-old woman with no breast cancer risk factors and no history of breast surgery had a screening mammogram (Figure 8). Left mammogram shows the breast heterogeneously dense, which may obscure small masses, and it can identify medium density SDD (red arrow) at the cranio-caudal (CC) mammogram view (a) and medio-lateral oblique (MLO) mammogram view (b). Targeted US shows intraductal mass (c) and high elastography (d); H\&E staining (x10) shows fibroadenoma (e). Fibroadenoma is the most common benign mass in women's breasts. There is no specific localization in the breast and the follow-up generally is sufficient.

\section{Discussion}

In mammography, the finding of a solitary dilated duct is described when a tubular or branched structure is identified in the retro-areolar region of the breast. In this series of cases, only cases of SDD, regardless of its density or thickness, and without associated findings, were presented. In the presence of a suspicious finding, SDD may be secondary to the main finding, which goes against the BI-RADS ${ }^{\circledR}$ description. Targeted US performed to the finding of SDD revealed different ultrasound findings for similar lesions on mammography (SDD), as in the cases presented below.

This case series demonstrates the heterogeneity presentations of SDD when targeted ultrasound is performed. In all these cases, the histological investigation results in benign lesions and all patients were asymptomatic. This finding had been described by Choudhery et al who found no malignancy associated with SDD in asymptomatic women. ${ }^{8}$ In another study that evaluated dilated ducts by ultrasound, when evaluating asymptomatic patients, only one case out of 66 had a malignancy result, but not all cases had SDD findings on mammography. ${ }^{9}$

Few studies assess the solitary dilated duct visualized by mammography with targeted ultrasound, and when this happens, it was not in all cases. A paper described 46 cases with asymmetric dilated ducts on mammography, but only seven were submitted to ultrasound. One mass with malignant results was identified and only ductal ectasia was found in the other six cases. Most of the lesions were benign (35/46) and papilloma was the most relevant finding (10/35). ${ }^{10}$ Similar to Chang et al, who identified 21 SDDs in 264,476 retrospective examinations of mammograms of which ultrasound was performed in only 14 cases. Of this, seven had core needle biopsy performed and only one resulted in ductal carcinoma in situ and six fibrocystic changes. ${ }^{3}$ 

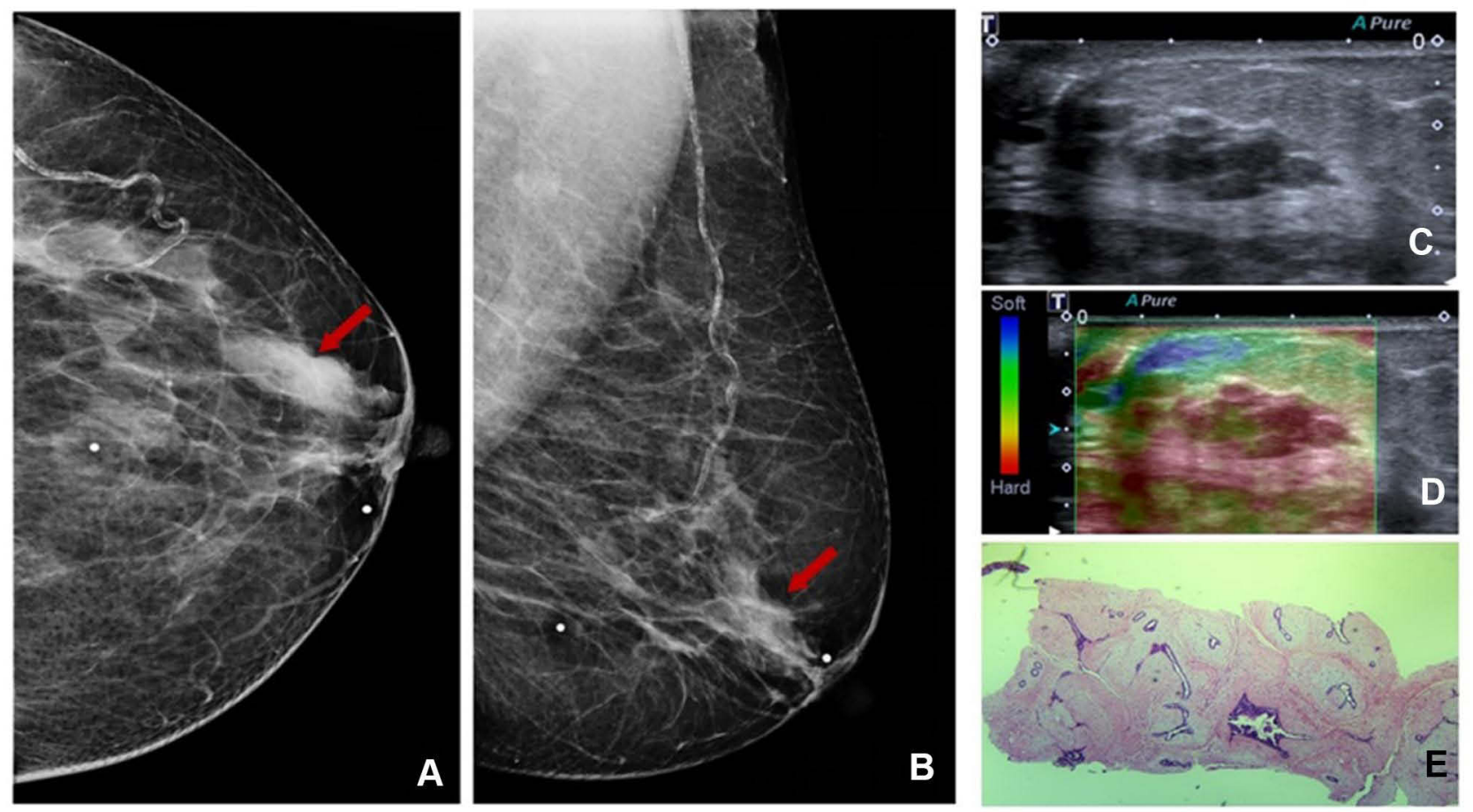

Figure 8 Left mammogram SDD (red arrow) at cranio-caudal (CC) (A) and medio-lateral oblique (MLO) (B) mammogram view. Targeted US shows intraductal mass (C) and high elastography (D); H\&E staining ( $x \mid 0)$ shows fibroadenoma (E).

\section{Conclusion}

A solitary dilated duct visualized by mammography is a rare finding and is poorly common be related to malignancy. Targeted US provides additional information, which can help in the decision to perform core needle biopsy and reduce false-positive results of SDD visualized by mammography.

\section{Data Sharing Statement}

The original data used as a source for this paper can be accessed at ClinicalTrials.gov (NCT03161392).

\section{Ethical Approval}

This paper has the approval of the local ethic committee.

All patients accepted the informed consent form.

\section{Acknowledgment}

Part of this pictorial essay was presented at the European Congress of Radiology in 2019 in the format of a poster, access link: https://epos.myesr.org/poster/esr/ecr2019/C-1663

\section{Funding}

There is no funding to report.

\section{Disclosure}

The authors declare no conflicts of interest.

\section{References}

1. D’Orsi CJ, Ea S, Eb M, Ea M. ACR BI-RADS Atlas, breast imaging reporting and data system. Reston, VA: American College of Radiology; 2013.

2. D'Orsi CJ, Eb M, Ikeda DM, editors. Breast imaging reporting and data system: ACR BI-RADS - breast imaging atlas-4th edition. Reston, VA: American College of Radiology; 2003.

3. Chang CB, Lvoff NM, Leung JW, et al. Solitary dilated duct identified at mammography: outcomes analysis. AJR Am J Roentgenol. 2010;194 (2):378-382. doi:10.2214/AJR.09.2944

4. Ayres VJ, Ramalho LC, Fernandes CE, Fleury EFC, Pompei LM. Solitary dilated duct visualised by mammography: ultrasound and anatomopathological correlation. Clin Radiol. 2020;75(12):962.e9-. e15. doi:10.1016/j.crad.2020.08.012

5. Ferris-James DM, Iuanow E, Mehta TS, Shaheen RM, Slanetz PJ. Imaging approaches to diagnosis and management of common ductal abnormalities. Radiographics. 2012;32(4):1009-1030. doi:10.1148/ rg. 324115150

6. Boufelli G, Giannotti MA, Ruiz CA, et al. Papillomas of the breast: factors associated with underestimation. Eur J Cancer Prev. 2017;27 (4):310.

7. Fleury EFC, Gianini AC, Marcomini K, Oliveira V. The feasibility of classifying breast masses using a computer-assisted diagnosis (CAD) system based on ultrasound elastography and BI-RADS Lexicon. Technol Cancer Res Treat. 2018;17:1533033818763461. doi:10.1177/ 1533033818763461 
8. Choudhery S, Simmons C, Woodard GA, Bhatt AA, Anderson TL, Lee CU. Outcomes of solitary dilated breast ducts in symptomatic and asymptomatic patients. Br J Radiol. 2020;93(1109):20191039. doi:10.1259/bjr.20191039

9. Song SE, Yie A, Seo BK, et al. A prospective study about abnormal ductal dilatations without associated masses on breast US: what is the significance for us? Acad Radiol. 2012;19(3):296-302. doi:10.1016/j. acra.2011.10.021
10. Huynh PT, Parellada JA, de Paredes ES, et al. Dilated duct pattern at mammography. Radiology. 1997;204(1):137-141. doi:10.1148/ radiology.204.1.9205235

\section{Publish your work in this journal}

Breast Cancer - Targets and Therapy is an international, peer-reviewed open access journal focusing on breast cancer research, identification of therapeutic targets and the optimal use of preventative and integrated treatment interventions to achieve improved outcomes, enhanced survival and quality of life for the cancer patient.
The manuscript management system is completely online and includes a very quick and fair peer-review system, which is all easy to use. Visit http://www.dovepress.com/testimonials.php to read real quotes from published authors. 